\title{
Oxidative stress biomarkers in evaluation of therapeutic potential of Ascorbic acidin poultry under hot Climate
}

\author{
Sandeep Sodhi Kakkar ${ }^{1}$, H .S. Banga, R.S .Brar \\ ${ }^{I}$ Department of Vety Physiology and Biochemistry \\ Department of Vety Pathology GADVASU Ludhiana (India) \\ Corresponding Author: H.S. Banga
}

\begin{abstract}
Reactive oxygen species (ROS) are a type of oxygen-derived free radicals which are produced normally in cells during mitochondrial respiration and energy generation, but they are degraded and removed by cellular defense systems. When the production of ROS increases or the scavenging systems are ineffective, the result is an excess of these free radicals, leading to a condition called oxidative stress. ROS damage DNA, biomembrane lipids, proteins and other macromolecules. These phenomena contribute to the development of several metabolic dysfunctions, including cell death by causing "oxidative stress" and "oxidative damage.The detrimental effects of high ambient temperature on broiler performance have been widely documented. Feed consumption and growth rate decrease at high ambient temperature. Antioxidant status of the organism is depleted as result of heat induced oxidative stress The degree of lipid peroxidation is used as an indicator of ROS mediated damage and the concentration of MDA in blood and tissues are generally used as biomarkers of oxidative stress. In addition analyses of the activity of the enzymes glutathione peroxidase, catalase and superoxide dismutase are important in determining whether oxidative stress reactions are induced in cells and which cells or organs have been damaged. Antioxidants like ascorbic acid are free radical quenchers, and thereforealleviate the negative effect of heat stress and may stimulate the biosynthesis and secretion of antioxidant enzymes which scavenge the free radicals.
\end{abstract}

Keywords: Heat Stress, Poultry, Antioxidants, Oxidative Stress

\section{Introduction}

\section{Reactive oxygen species (ROS) and cell damage}

The reduction-oxidation (redox) reactions that occur during normal mitochondrial metabolism duringnormal respiration, for example, molecular oxygen is sequentially reduced in mitochondria by the addition offour electrons to generate water. In the process, small amounts of toxic intermediate species ROS are generated bypartial reduction of oxygen; these include superoxide radicals $(\mathrm{O} 2 \cdot \square)$, hydrogen peroxide $(\mathrm{H} 2 \mathrm{O} 2)$, and $\mathrm{OH} \bullet$. A disturbance in the intracellular balance between ROS production and the activities of the anti-oxidantdefense systems in favor of the former renders an oxidatively stressed cell. Reactive oxygen species can attack vital cell components like polyunsaturated fatty acids, proteins, and nucleic acids. To a lesser extent, carbohydrates are also the targets of ROS. These reactions can alter intrinsic membrane properties like fluidity, ion transport, loss of enzyme activity, protein cross-linking, inhibition of protein synthesis, DNA damage: ultimately resulting in cell death. Some of the well-known consequences of generation of the free radicals in vivo are: DNA strand scission, nucleic acid base modification, protein oxidation and lipidperoxidation (Bandyopadhyay, 1999; Caden and Davies, 2000).

- Lipid peroxidation of membranes: Double bonds in membrane polyunsaturated lipids are vulnerable toattack by oxygen-derived free radicals. The lipid radical interactions yield peroxides, which are themselvesunstable and reactive, and an autocatalytic chain reaction initiates.

- Cross-linking of proteins: Free radicals promote sulfhydryl-mediated protein cross-linking, resulting inenhanced degradation or loss of enzymatic activity.Free-radical reactions may also directly cause polypeptidefragmentation.

- DNA fragmentation: Free-radical reactions with thymine in nuclear and mitochondrial DNA producesinglestrand breaks. Such DNA damage has been implicated in cell death, aging, and malignant transformationof cells species.

\section{Biochemical Markers of Oxidative Stress}

Analyses of the activity of enzymes are important in determining whether oxidative stress reactions are induced in cells and which cells or organs have been damaged(Ognik and Krauze,2016).Activity of antioxidant 
enzymes such as superoxide dismutases (SOD) glutathione peroxidase (GPX) and catalase have been quantified in plasma as measures of antioxidant capabilities). Measurement of the MDA content (liver and serum) and the Antioxidant enzymes (liver and serum) are investigated as Biochemical markers of Oxidative stress induced by aging (Rizvi and Maurya, 2007) and heat stress (Altan et. al., 2003; Lin, 2008).

\section{MDA conc:}

The degree of lipid peroxidation is often used as an indicator of ROS mediated damage. The concentration of MDA in blood and tissues are generally used as biomarkers of lipid peroxidation. MDA is typically quantified from blood samples with the most popular method being a colorimetric assay based on the reaction between MDA and thiobarbituric acid (TBA) (Meagher and FitzGerald, 2000).However, although suitable for high throughput analysis, this TBA reacting substances (TBARS) assay lacks specificity for MDA, with aldehydes other than MDA reacting with TBA to produce compounds that absorb in the same range as MDA(KUHNand Borchert,2002). Heat stress also caused oxidative stress, increased red blood cell susceptibility to peroxidation, as indicated by increased MDA concentration in our studies (Banga et al, 2009)

\section{Superoxide dismutases:}

The first enzyme involved in the antioxidant defense is the superoxide dismutase: a metalloprotein .In eukaryotic cells, the predominant forms are the copper-containing enzyme and the zinc-containing enzyme, located in the cytosol. The second type is the manganesecontainingSOD found in the mitochondrial matrix4. The biosynthesis of SOD is mainly controlled by its substrate, the $\mathrm{O} 2$-The rate of spontaneous decay of superoxide is significantlyincreased by the action of superoxide dismutases(SODs) found in many cell types .

\section{Glutathione peroxidase:}

Glutathione peroxidase 26 catalyses the reaction of hydroperoxides with reduced glutathione (GSH) to form glutathione disulphide (GSSG) and the reduction product of the hydroperoxide6 (Figure 2). This enzyme is specific for its hydrogen donor, GSH, and nonspecific for the hydroperoxides ranging from $\mathrm{H} 2 \mathrm{O} 2$ to organic hydroperoxides. It is a seleno-enzyme; two-third of which (in liver) is present in the cytosol and one-third in the mitochondria.

\section{Catalase:}

Catalase present in almost all the mammalian cells is localized in the peroxisomes or the microperoxisomes6. It is a hemoprotein and catalyses the decomposition of $\mathrm{H} 2 \mathrm{O} 2$ to water and oxygen and thus protects the cell from oxidative damage by $\mathrm{H} 2 \mathrm{O} 2$ and. $\mathrm{OH}$.

\section{Glutathione:}

Reduced glutathione (GSH) is a major intracellular non-protein -SH compound and is accepted as the most important intracellular hydrophilic antioxidant (Wu et al, 2004) GSH has many biological functions, including nutrient metabolism, and regulation of cellular events such as gene expression, DNA and protein synthesis, cell proliferation and apoptosis, signal transduction, cytokine production and immune response, and protein glutathionylation. GSH also plays a role in maintenance of membrane protein -SH groups in the reduced form, the oxidation of which can otherwise cause altered cellular structure and function. Different studies have pointed out the importance of determination of blood glutathione for both pathological and physiological purposes.Studies have reveled that the reduced glutathione is important markers of oxidative stress.

\section{Heat induced oxidative stress in poultry}

A hot environment is one of the important stressors in poultry production associated with economic losses to the poultry industry in the hotter regions of the world. It causes poor growth performance (Bottje and Harrison, 1985) and immunosuppression (Young, 1990).Elevated environmental temperature causes disorders of the body-heat regulating mechanism in poultry (Miyazava, 2007). When the temperature exceeds $30{ }^{\circ} \mathrm{C}$, signs of heat stress are likely to appear which affects egg production and egg shell quality, decreases feed consumption and live weight, and disrupts the acid-base balance of the blood, thus causing some changes in metabolism and oxidative damage to cells and high mortality (Yahav et al., 1995). Mujahid et al. (2005, 2007) observed significantly enhanced superoxide anion production in heat stress-treated skeletal muscle mitochondria of meattype chicken. Effect of heat stress $(38+/-1$ degree $\mathrm{C}$ for $3 \mathrm{~h})$ on oxidative stress, lipid peroxidation and some stress parameters in commercial broilers showed a significant alterations for MDA concentration. CAT, SOD and GPx activities(Lin et.al.2006a).Serum and liver lipid peroxidation levels were found to be significantly (P < 0.05 ) higher in chickens that were exposed to heat stress $(40+/-1$ degrees $C$ and relative humidity of $80+/-$ $5 \%$ in an environmental chamber) for $4 \mathrm{~h}$ daily for 5 or 10 days (Ramnath et al., 2008) ). The resultant heat stress comes from the interactions among air temperature, humidity, radiant heat and air speed, where the air 
temperature plays the major role (Lin et al 2006b). Statistically significant $(\mathrm{P} \leq 0.05)$ increases in plasma malondialdehyde (MDA), erythrocyte MDA, glutathione peroxidase (GSH-Px), catalase (CAT), superoxide dismutase (SOD), and egg yolk MDA concentration, and a decrease in plasma vitamin E were seen in the experimental group during heat stress. Egg quality parameters also decreased in the experimental group during heat stress(Yardebi and Turkay ,2008). Tan(2010) in his extensive studies studied observed significant differences in the activities of SOD and GSH-Px in serum and liver between the control and the groups exposed to high ambient temperature $\left(32,35\right.$, and $38^{\circ} \mathrm{C}$ ) in 6 -wk-old broiler chickens for $3 \mathrm{~h}$, and the activity of SOD and GSH-Px rose in a temperaturedependent manner The activity of CAT in the liver and serum also showed a temperature-dependent increasing trend, Thus the results from various studies demonstrate that exposure to high ambient temperature can cause a compensatory increase in the activity of antioxidative enzymes.

\section{Therapeutic Potential of antioxidants}

It has been stated that, under normal physiological conditions, the balance between prooxidant and antioxidant compounds moderately favors prooxidants, thus engendering a slight oxidative stress,requiring the intervention of endogenous antioxidant systems of the organism. These systems present in aqueous and membrane (Aurelia,2015).The first identified types of antioxidant defense systems developed against oxidative damage, are those that prevent reactive oxygen species occurrence and those that block, capture, radicals thatare formedcell compartments can be enzymatic and nonenzymatic(Puthpongsiripornet.al,2001) Thus, the antioxidant supplementsmay improve the organism's capacity to contain the oxidativestress, that cannot be amended by the intervention ofendogenous antioxidant defenses(Packer et.al.1979).

Therefore enhancing ROS detoxifying capacity remains the primary target in heat stressed broilers, which can be achieved either by scavenging ROS, inhibiting enzymatic processes that lead to the formation of ROS or by upregulating and protecting endogenous antioxidant defenses. As alleviation of the negative effect of high environmental temperature through cooling of animal house is impractical and costly, therefore workers devoted to dietary manipulation of heat induced oxidative stress (Yahav,2009). Stress is a factor that has been shown to increase the requirement for vitamins and energy.

Sahin et al. (2003) showed that chronic exposure to high temperature (10-day-old Japanese quails were exposed to $34{ }^{\circ} \mathrm{C}$ for $8 \mathrm{~h} / \mathrm{d}$ followed by $22{ }^{\circ} \mathrm{C}$ for $32 \mathrm{~d}$ ) decreased serum ascorbic and tocopherol concentrations by $40 \%$ and $29 \%$. This supports the contention that the components of the animal's antioxidant system become depletedfollowing chronic HS andanimal's antioxidant system needs to be re-enforced. Ascorbic acid, one of the most ubiquitous hydrosoluble antioxidants., Vitamin C scavenges hydroxyl, alkoxyl and superoxide radical anion in biological media, but also reactive nitrogenated species, by forming semidehydroascorbic acid and therefore, prevents theoxidative decay of essential biomolecules (Frei,1989). Ascorbic acid participates in numerous biochemical reactions The Lenantiomer of ascorbic acid (vitamin C) is involved in maintaining vascular and connective tissue's integrity, in iron absorbtion and collagen biosynthesis, neuroprotection, but also in hematopoiesisand leukocyte functioning . Vitamin C accomplishes essential roles in the brain, including being cofactor of dopamine beta-hydroxylase, and thus takes part in catecholamine biosynthesis.It also protects membrane phospholipids from peroxidative damage, and was demonstrated to be an efficient free radical scavenger in the brain . Ascorbic acid can be synthesized by poultry and it is not required to be supplemented in the diet under normal conditions. When birds are challenged with stressors, however, the supplementation of AA might be beneficial for the performance of broilers (Pardue and Thaxton, 1982)

Uses of Vitamin $\mathrm{C}$ (ascorbic acid) as feed additive was aimed at reducing the heat stress in birds(ElHabbak et.al.2011).The significant increase of CAT, GST and SOD activities in ascorbic acid treated birds may be considered as a protective mechanism against oxidative stress and lipid peroxidation ( (Panda et al., 2008). The significant reduction of MDA in ascorbic acid treated birds was observed (Nagra ,2006)in broiler chicken. Vitamin C supplementation increased the antioxidant defense and ameliorate the oxidative stress of heat stressed in poultry (Khan et al., 2012). This may suggest potentiality of ascorbic acid for stimulation of antioxidants enzymes biosynthesis to ameliorate heat induced oxidative damage. However, further investigation at the molecular level is required in the future studies. Heat exposure affects poultry production on a worldwide basis and has a significant impact on well-being and production. Heat stress (HS) occurs when the amount of heat produced by an animal surpasses the animal's capacity to dissipate the heat to its surrounding environment. This imbalance may be caused by variations in a combination of environmental factors (e.g. sunlight, thermal irradiation, air temperature, humidity, and movement) and characteristics of the animal (e.g. species, gender, and rate of metabolism).Current review strongly suggests that HS induces oxidative stress in poultry. The alleviation of oxidative damage can be approached though nutritional strategies. It is repeatedy reported that ascorbic acid supplementation is beneficial to heat stressed poultry. This review supports the contention that antioxidants may have potential in challenging conditions. The complex effects of oxidative stress in HS highlight the importance of identifying the biomarker that has maximum specificity for pathophysiological effects in the relevant compartments for it to be useful for individualization of treatment strategies. 


\section{References}

[1] Aurelia M. P, Aneta P(2015) ,"The role of antioxidants in the chemistry of oxidative stress: A review”European Journal of Medicinal Chemistry, Vol. 97 pp 55-74

[2] Altan, O., A. Pabuccuoglu, A. Altan, S. Konyalioglu and H. Bayraktar,( 2003) "Effect of heat stress on oxidative stress, lipid peroxidation and some stress parameters in broilers." Br. Poult. Sci., Vol44, pp545-550.

[3] Bandyopadhyay Uday, Dipak Das and Ranajit K. Banerjee, (1999)"Reactive oxygen species: Oxidative damageand pathogenesis"current science, vol. 77, NO. 5,pp 658-666

[4] Banga, H.S., Sodhi, S. and Brar, R.S. (2009), "Biochemical and Histopathological changes in chicks with Hyperthermia", Indian Vet J vol 8pp 638-639

[5] Bottje, W. G., and P. C. Harrison,( 1985)., “ Effect of carbonated water on growth performance of cockerels subjected to constant and cyclic heat stress temperatures”. Poult. Sci. vol 64pp1285-1292.

[6] Cadenas, E. and K.J.A. Davies,( 2000) "Mitochondrial free radical generation, oxidative stress and aging". Free Radic. Biol. Med., vol 29pp 222-230.

[7] El-Habbak, M.M., A.A. El-Ghamry, G.M. El-Mallah, H.H. Younis and E.M. El-Komy,( 2011)“ Influence of dietary Vitamin E and C supplementation on performance and some metabolic response of broiler chicks subjected to heat stress". World J. Agric. Sci., vol7pp258-269.

[8] Frei B, England L, Ames BN,( 1989), “Ascorbate is an outstanding antioxidant in human blood plasma”. Proc Natl Acad Sci USA,vol86pp 6377-6381

[9] Khan RU, Naz S, Nikousefat Z, Selvaggi M, Laudadio V, Tufarelli V." Effect of ascorbic acid in heat-stressed poultry”. World's Poult Sci J. 2012;68:477-489

[10] Kuhn, H. and A. Borchert,( 2002),“Regulation of enzymatic lipid peroxidation: The interplay of peroxidizing and peroxide reducing enzymes”. Free Radical Biol. Med.,vol 33pp 154-172.

[11] Lin H, Decuypere E, Buyse J,(2006a), “Acute heat stress induces oxidative stress in broiler chickens”. Comp Biochem Physiol Part A: Mol \& Integr Physiol. Vol,14pp11-17

[12] Lin H, Jiao HC, Buyse J, Decuypere E.,(2006b ), “Strategies for preventing heat stress in poultry”. World's Poult Sci J. vol62pp7186

[13] Lin, H., D. De Vos, E. Decuypere, and J. Buyse. (2008)“ Dynamic changes in parameters of redox balance after mild heat stress in aged laying hens (Gallus gallus domesticus)”. Comp. Biochem. Physiol. C Toxicol. Pharmacol.vol 147 pp30-35.

[14] Meagher, E.A.and G.A. FitzGerald,(2000), "Indices of lipid peroxidation in vivo: strengths and limitations Free Radical Biology and Medicine",vol 28 pp 1745-1750

[15] Mujahid, A., Y. Akiba, C. H. Warden, and M. Toyomizu,(2007a)" Sequential changes in superoxide production, anion carriers and substrate oxidation in skeletal muscle mitochondria of heatstressed chickens”. FEBS Lett.vol 581pp3461-3467.

[16] Mujahid, A., N. R. Pumford, W. Bottje, K. Nakagawa, T. Miyazawa, Y. Akiba, and M. Toyomizu ,(2007b)“ Mitochondrial oxidative damage in chicken skeletal muscle induced by acute heat stress". Jpn. Poult. Sci.vol 44pp439-445

[17] Mujahid, A., Y. Yoshiki, Y. Akiba and M. Toyomizu, (2005), "Superoxide radical production in chicken skeletal muscle induced by acute heat stress". Poult. Sci., vol 84pp 307-314.

[18] Ognik K. and M. Krauze,(2016)“The potential for using enzymatic assays to assess the health of turkeys”,World's Poult Sci JVol 72, (3) pp. $535-55$

[19] Nagra, S. Sodhi and Y. Singh (2006)“ Efficacy of herbal and synthetic ascorbic acid supplementation in broiler chicks during heat stress”. Ind. J. Anim. Nutr,vol 23(4)pp 247-249.

[20] Packer JE, Slater TF, Wilson RL,(1979)“ Direct observation of a free radical interaction between vitamin E and vitamin C", Nature. vol278pp737-738

[21] Panda, A.K., S.V. Ramarao, M.V.L.N. Raju and R.N. Chatterjee, (2008), "Effect of dietary supplementation with vitamins E and C on production performance, immune responses and antioxidant status of White Leghorn layers under tropical summer conditions". Br. Poult. Sci., vol49pp592-599.

[22] Pardue, S.l. and Thaxton, J.P. ,(1982)“ Enhanced livability and improved immunological responsiveness in ascorbic acid supplemented cockerels during acute heat stress". Poultry Science vol61pp1522

[23] Puthpongsiriporn, U., S.E. Scheideler, J.L. Sell and M.M. Beck, (2001), "Effects of vitamin E and C supplementation on performance, In vitro lymphocyte proliferation and antioxidant status of laying hens during heat stress". Poult. Sci.,vol 80pp 11901200 .

[24] Ramnath, V., P.S. Rekha and K.S. Sujatha, (2008)“"Amelioration of heat stress induced disturbances of antioxidant defense system in chicken by Brahma Rasayana. Evidence-Based Complement”. Altern. Med.,vol 5pp 77-84

[25] Rizvi SI, Maurya PK,(2007), "Markers of oxidative stress in erythrocytes during aging in humans". Ann N Y Acad Sci. vol1100pp373-382

[26] Sahin, K., M. Onderci, N. Sahin, M.F. Gursu and O. Kucuk, (2003), "Dietary vitamin C and folic acid supplementation ameliorates the detrimental effects of heat stress in Japanese quail”. J. Nutr.,vol 133pp 1882-188

[27] Tan GY, Yang L, Fu YQ, Feng JH, Zhang MH.,(2010)“Effects of different acute high ambient temperatures on function of hepatic mitochondrial respiration, antioxidative enzymes, and oxidative injury in broiler chickens". Poult Sci. vol,89pp115-122

[28] Wu G, Fang YZ, Yang S, Lupton JR, Turner ND,(2004) "Glutathione metabolism and its implications for health". J Nutr. vol134pp489-492

[29] Yahav, S., S. Goldfeld, I. Plavnik, and S. Hurwitz,(1995), “ Physiological responses of chickens and turkeys to relative humidity during exposure to high ambient temperature". J. Therm. Biol. vol20pp245-253.

[30] Yahav S.(2009)“ Alleviating heat stress in domestic fowl: different strategies”. World's Poult Sci J. ,vol65pp719-732

[31] Yardibi, H. and G. Turkay,( 2008) "The effects of vitamin E on the antioxidant system, egg production and egg quality in heat stressed laying hens". Turk. J. Anim. Sci., vol32pp 319-325

[32] Young, R. A.,( 1990)“ Stress proteins and immunology”. Annu. Rev. Immunol. Vol 8 pp401-420.

Sandeep Sodhi Kakkar. "Oxidative stress biomarkers in evaluation of therapeutic potential of Ascorbic acidin poultry under hot Climate." IOSR Journal of Agriculture and Veterinary Science (IOSR-JAVS) 10.7 (2017): 41-44. 